\title{
Malignant germ cell tumors and sex cord-stromal tumor clinical experience in a tertiary spanish hospital
}

\author{
A. Coves, R. Oliver, G. López, JM. Seoane, C. Álvarez, A. Tejerizo
}

\section{Introduction}

Non-epithelial ovarian tumors account for approximately $10 \%$ of all ovarian cancer. Malignant germ cell tumors usually develop in young females in whom fertility is important. Granulose cell tumor, the most common type of sex cord-stromal tumor, occurs more often in middle age and postmenopausal women.

The aim of this study is to describe the clinico-pathological features and treatment of non-epithelial ovarian tumors in a Spanish tertiary hospital.

\section{Material and methods}

Observational retrospective study. Data from 25 patients with non epithelial ovarian cancer were collected between 1999-2017 in "12 de Octubre" hospital, Madrid, Spain. Twenty (80\%) were germ cell tumors and five (20\%) were sex cord-stromal tumors.

\section{Results}

Mean age at diagnosis was 27 years (range 10-75). Mean age was 22, 18, 22, 30 and 52 for immature teratoma, dysgerminoma, yolk sac tumor, embryonal carcinoma and granulose cell tumors, respectively.

Information of clinical presentation was available in 11 patients. Five of these presented abdominal pain and abdominal distension. One presented with acute abdomen because of torsion of the tumor. Another one had obstructive uropathy. Four patients were incidental diagnosis, two in cesarean context and the others in postmenopausal bleeding study context.

Surgery was the first treatment for $23(92 \%)$ patients. Only $2(8 \%)$ received neoadjuvant chemotherapy (1 dysgerminoma IIB, 1 granulose cell tumors IIIC).

Most of the patients, 19 (76\%) underwent fertility sparing surgery. Six (24\%) patients underwent radical surgery: 3 postmenopausal patients; 1 who had already completed her child-bearing, 1 with previous anexectomy because of tuboovaric abcess, and 11 years old patient, with dysgerminoma stage IIB disease.

Median follow-up period was 125 months (range: 23-225 months).

Recurrence disease occurred in $4(16 \%)$ patients: 1 out of 6 patients (16\%) affected by granulose cell tumors stage IC, in 2 out of $10(20 \%)$ with immature teratoma stage IIIC and 1 out of $6(16 \%)$ patients with dysgerminoma stage IA disease with thoracic recurrence.

Two (8\%) patients died. One of them because of the tumor (dysgerminoma stage IA with thoracic recurrence). The other one due to unknown cause.
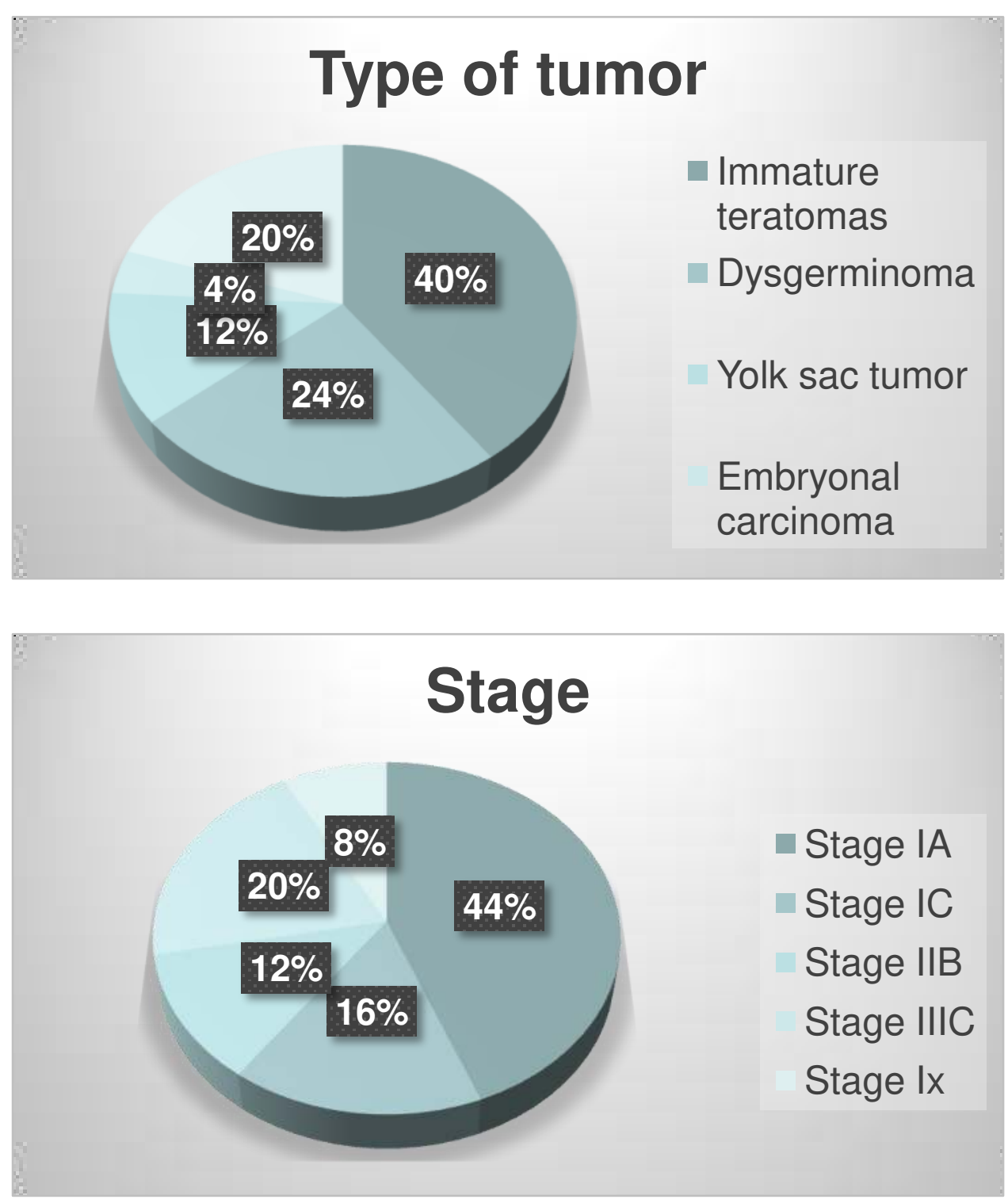

\section{Adjuvant chemotherapy}
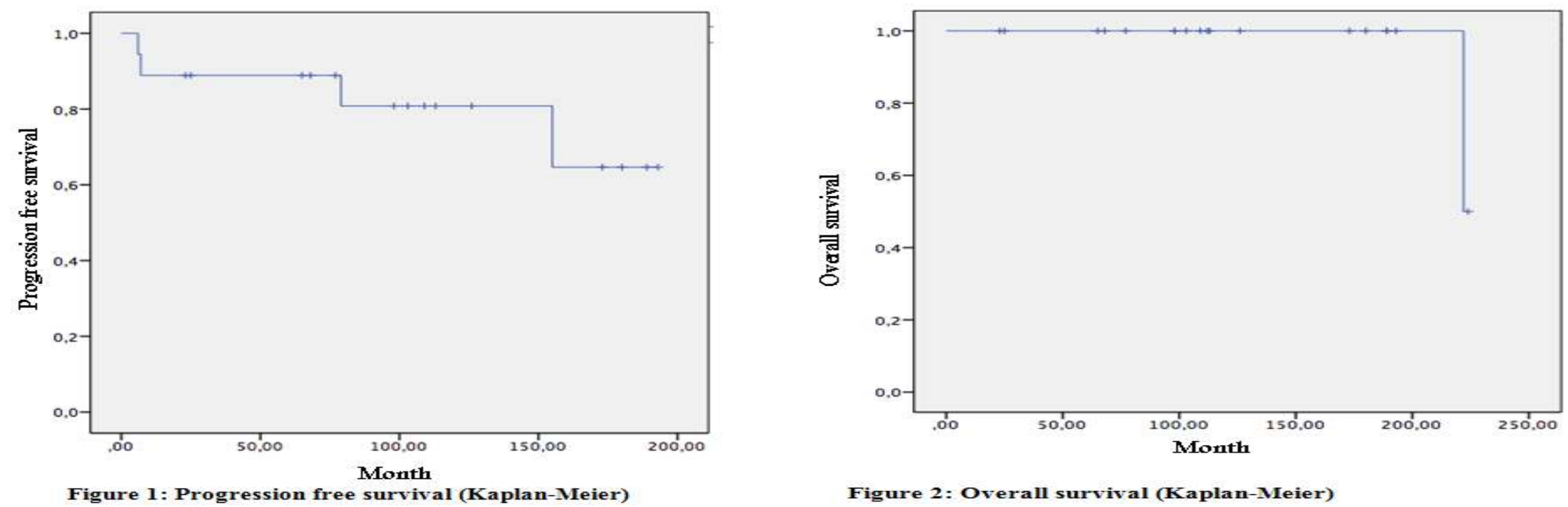

\section{Conclusion}

In our study most of non-epithelial ovarian tumors are diagnosed at an early stage (stage I in $60 \%$ ). Given the youth of patients (mean age at diagnosis 27$)$, fertility sparing surgery was the main treatment $(72 \%)$. This appears to be safe, with excellent survival after longterm follow-up.

Adjuvant chemotherapy is not always recommended, stage IA dysgerminoma, inmature teratoma and granulose cell tumors do not require further adjuvant chemotherapy. 